\title{
Improved pain management outcomes with continuous infusion of a local anesthetic after thoracotomy
}

\author{
Grayson H. Wheatley III, MD, ${ }^{\text {a }}$ David H. Rosenbaum, MD, ${ }^{a}$ Michelle C. Paul, BS, ${ }^{a}$ Alan P. Dine, BSN, \\ Michael A. Wait, MD, ${ }^{a}$ Dan M. Meyer, MD, ${ }^{a}$ Michael E. Jessen, MD, ${ }^{a}$ W. Steves Ring, MD, ${ }^{a}$ and \\ J. Michael DiMaio, MD
}

From the Department of Cardiovascular and Thoracic Surgery, ${ }^{a}$ University of Texas Southwestern Medical Center, Dallas, Tex,

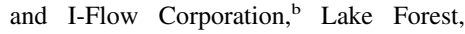
Calif.

Supported in part by the Donald W. Reynolds Cardiovascular Research Center and by the National Institutes of Health 5T32GM08593 Training Program in Burns, Trauma and Critical Care.

Presented at the annual meeting of the American College of Surgeons, Chicago, Ill, October 2003.

Received for publication Oct 22, 2004; revisions received Jan 20, 2005; accepted for publication Feb 10, 2005.

Address for reprints: J. Michael DiMaio, MD, University of Texas Southwestern Medical School, 5323 Harry Hines Blvd, Dallas, TX 75390-8879 (E-mail: michael. dimaio@utsouthwestern.edu).

J Thorac Cardiovasc Surg 2005;130:464-8

$0022-5223 / \$ 30.00$

Copyright $\odot 2005$ by The American Association for Thoracic Surgery

doi:10.1016/j.jtcvs.2005.02.011
Objective: We sought to determine the effectiveness of an incisional infusion of local anesthetics through a continuous-infusion elastomeric pump for the management of postoperative pain after thoracotomy.

Methods: We performed a retrospective comparative analysis of 110 patients undergoing thoracotomies between November 1999 and March 2003. Postoperative pain management with a continuous-infusion elastomeric pump providing local anesthetic into the incisional area was compared with a single-shot epidural in combination with continuous local anesthetic infusion and continuous thoracic epidural infusion. Data sources were reviewed for mean narcotic use, pain score, and complications.

Results: After thoracotomy procedures, 38 patients received the ON-Q Pain Relief System (I-Flow Corp, Lake Forest, Calif), 32 received the ON-Q device and single-shot epidural infusion, and 40 received continuous epidural infusion. Demographic attributes, including age, body mass index, and sex were similar between the groups. Preoperative American Society of Anesthesiologists status was significantly higher in the ON-Q group compared with that in the other groups $(P=.02)$. Narcotic use and pain scores were significantly reduced in the ON-Q group compared with that in the epidural group at all time points $(P<.001)$. There were no wound-healing complications or infections associated with the use of the pump.

Conclusion: A continuous infusion of $0.25 \%$ bupivacaine at $4 \mathrm{~mL} / \mathrm{h}$ through the ON-Q elastomeric infusion pump is a safe and effective adjunct in postoperative pain management after thoracotomy. The use of the ON-Q Pain Relief System results in decreased narcotic use and lower pain scores compared with continuous epidural infusion.

$\mathrm{P}$ osterolateral thoracotomy has been described as one of the most painful surgical procedures because of the extent of surgical insult. Inadequate pain control after thoracic procedures can result in increased morbidity, length of stay, and delay in overall recovery. ${ }^{1-3}$ In particular, inadequate pain control might lead to postoperative pulmonary complications by reducing patient compliance with pulmonary physiotherapy and inhibiting diaphragmatic function through spinal reflex inhibition of phrenic nerve activity. ${ }^{4}$ Importantly, control of postoperative pain after thoracotomy has been demonstrated to decrease the incidence of postoperative pulmonary complications, such as mucous plugging, hypoxia, atelectasis, and pulmonary infections. ${ }^{5,6}$ The problems associated with restriction of chest wall motion and shallow breathing have been shown to be accentuated in the obese, the elderly, patients who smoke, and those with existing pulmonary infections. ${ }^{7}$ Standard management of postthoracotomy pain has been the use of continuous or single-shot epidural infusions, patient-controlled analgesia (PCA), or a combination 
of both. As an alternative to epidural and PCA pain management, local anesthetics have been used to control pain at the site of incision or as a regional nerve block. The use of incisional local anesthetics for postoperative pain relief has been shown to diminish pain scores and use of supplementary analgesics. ${ }^{8,9}$ Unfortunately, the analgesic duration of most local anesthetics is relatively brief ( $<4$ hours) and might not provide significant long-term benefits with regard to analgesic or other patient-related outcomes (eg, length of stay and quality of life). Because of the difficulty of providing adequate postoperative pain relief, an approach that requires little or no intervention from the medical staff, nursing staff, or the patient, as well as one that is not dependent on narcotics, is considered to be of great interest to the medical community.

The ON-Q Pain Relief System (I-Flow Corp, Lake Forest, Calif) consists of an elastomeric pump that holds 270 $\mathrm{mL}$ of local anesthetic. The pump is connected by a flowlimiting valve to a small flexible catheter that acts as a soaker hose and allows continuous infusion of the drug to nearby tissues. The objective of this study was to examine the use of a continuous incisional infusion of local anesthetic as part of a multimodal approach to the management of postoperative pain after thoracotomy.

\section{Methods}

After institutional review board approval, a retrospective comparative analysis of 110 patients undergoing thoracotomies between November 1999 and March 2003 was performed at St Paul University Hospital, which is the main private referral hospital of the University of Texas Southwestern Medical Center. For inclusion in the study, patients were identified through the surgical department's database. A total of 198 thoracotomies were performed during this period at St Paul University Hospital. One hundred ten of these patients were included in this study. Seventy patients received the ON-Q Pain Relief System. Of these 70 patients, 38 received $\mathrm{ON}-\mathrm{Q}$ and $\mathrm{PCA}(\mathrm{ON}-\mathrm{Q})$. The remaining 32 received ON-Q, a single-shot epidural, and PCA (SSD group). For comparison, an additional 40 patients who received continuous infusion of medication through an epidural catheter (continuous epidural infusion [CEI] group) after thoracotomy were selected and matched for age, sex, and body mass index.

Three methods of postoperative pain management were included in the review. One group received the ON-Q Pain Relief System (I-Flow Corp, Lake Forest, Calif) filled with $270 \mathrm{~mL}$ of $0.25 \%$ bupivacaine infusing at $4 \mathrm{~mL} / \mathrm{h}$ through two 20 -gauge Soaker catheters (I-Flow Corp), at $2 \mathrm{~mL} / \mathrm{h}$ each for 72 hours (ON-Q group). The second group consisted of single-shot epidural perioperatively with Duramorph (Elkins-Sinns, Inc, Cherry Hill, $\mathrm{NJ}$ ) in combination with ON-Q for postoperative pain management (SSD group). The third group received CEIs of bupivacaine alone or in combination with fentanyl or morphine (CEI group).

All patients underwent thoracotomies for various procedures. After completion of the procedure, our standard approach to the placement of the ON-Q Soaker catheters involves introduction through a peel-away trocar 3 to $5 \mathrm{~cm}$ anterior and superior to the incision. One catheter is placed at the level of the pericostal sutures adjacent to the intercostal nerve bundle, and the other is placed above the fascia in the subcutaneous space. A 5- to $10-\mathrm{mL}$ bolus of $0.25 \%$ bupivacaine without epinephrine is infused through the catheters just before the incisional site is closed. The catheters are then secured to the skin in a standard fashion with the tubing coiled and sewn to the skin to prevent accidental removal or kinking. The catheters are then attached to the pump and left in place for approximately 72 hours.

Detailed follow-up was abstracted from medical records recording narcotic use, visual analogue scale (VAS) pain score, time to first bowel movement, progression of diet to solid intake, American Society of Anesthesiologists status, and associated wound infection. VAS pain scores were obtained from detailed nursing notes, and mean daily scores were calculated for our analysis. These data were available for all patients.

Multiple groups were compared by 1-way analysis of variance tests to determine whether any of the population means differ from each other, with the Bonferroni post hoc test for multiple comparisons paired, the $\chi^{2}$ test, and the Student $t$ test as appropriate. Values are expressed as means \pm standard deviation.

\section{Results}

One hundred ten patients were included in this study. Thirtyeight patients received the ON-Q system, 32 patients received single-shot epidural plus the ON-Q system, and 40 patients received continuous epidural infusion for their primary postoperative pain management. Demographic variables of age, body mass index, and sex were similar among the groups. American Society of Anesthesiologists status at the beginning of the operation was statistically higher in the ON-Q group compared with that in both the CEI and singleshot epidural plus ON-Q groups $(P=.02)$. These demographic values are presented in Table 1 .

Mean and total narcotic use for the first 4 days postoperatively was significantly less in both the ON-Q and SSD groups compared with that in the CEI group $(P<.001)$, as seen in Figure 1.

Mean and overall VAS pain scores for the first 4 days postoperatively were significantly lower for both the ON-Q and SSD groups compared with the CEI group $(P<.001)$, as seen in Figure 2.

There were no statistically significant differences in narcotic use and pain scores between the ON-Q and SSD groups. Additionally, there were no differences noted in time to first bowel movement or progression to normal diet among the groups. There were no infections or any woundhealing complications noted in any patient receiving the ON-Q device.

\section{Discussion}

Pain is inadequately treated in about $75 \%$ of all surgical patients, which can result in increased morbidity, length of stay, and patient dissatisfaction. ${ }^{1-3,10,11}$ Painful experiences imprint themselves on the nervous system, intensifying the 
TABLE 1. Demographic characteristics of patients who received postoperative analgesia for thoracotomy according to analgesia type

\begin{tabular}{|c|c|c|c|c|c|}
\hline Variables & $\mathrm{n}$ & $\begin{array}{c}\text { ON-0 and PCA (ON-0 group), } \\
n=38\end{array}$ & $\begin{array}{l}\text { ON-Q, single-shot epidural } \\
\text { with Duramorph and PCA } \\
\text { (SSD group), } n=32\end{array}$ & $\begin{array}{c}\text { Continuous epidural } \\
\text { infusion (CEI group), } \\
\qquad n=40\end{array}$ & $P$ value \\
\hline Mean age (y) & 59.2 & $58 \pm 16.9$ & $59.6 \pm 14.3$ & $60.2 \pm 15.0$ & NS \\
\hline \multicolumn{6}{|l|}{ Sex } \\
\hline Male & 61 & 23 & 18 & 20 & NS \\
\hline Female & 49 & 15 & 14 & 20 & NS \\
\hline Mean BMI & 25.1 & $24.2 \pm 5.2$ & $25.2 \pm 8.6$ & $26 \pm 5.22$ & NS \\
\hline Mean ASA & 2.9 & $3.2 \pm 0.58^{*}$ & $2.87 \pm 0.62$ & $2.84 \pm 0.68$ & $<.05$ \\
\hline
\end{tabular}

PCA, Patient-controlled analgesia; NS, not significant; BMI, body mass index; ASA, American Society of Anesthesiologists. *0ÑO group versus CEI and SSD groups, $P<.05$.

response to subsequent stimuli. This can produce chronic pain syndromes in adults after thoracotomy. ${ }^{12}$ It is therefore of paramount importance to provide adequate pain control after surgical intervention.

One method for perioperative and postoperative pain control includes preemptive administration of a local anesthetic, followed by administration of narcotics during the postoperative period. Opioids, such as morphine, are considered the standard of care for the management of postoperative pain; however, disadvantages of this approach include the difficulty in maintaining a constant therapeutic drug level and the adverse effects of opioids. Narcotic side effects include but are not limited to respiratory depression, nausea, vomiting, sedation, pruritus, and urinary retention. These effects can worsen patients' conditions, resulting in extended hospital stays and increased costs. To avoid these adverse events, physicians are reluctant to prescribe and nurses often fail to administer adequate doses of opioid analgesics, which can lead to inadequate pain control. ${ }^{13,14}$

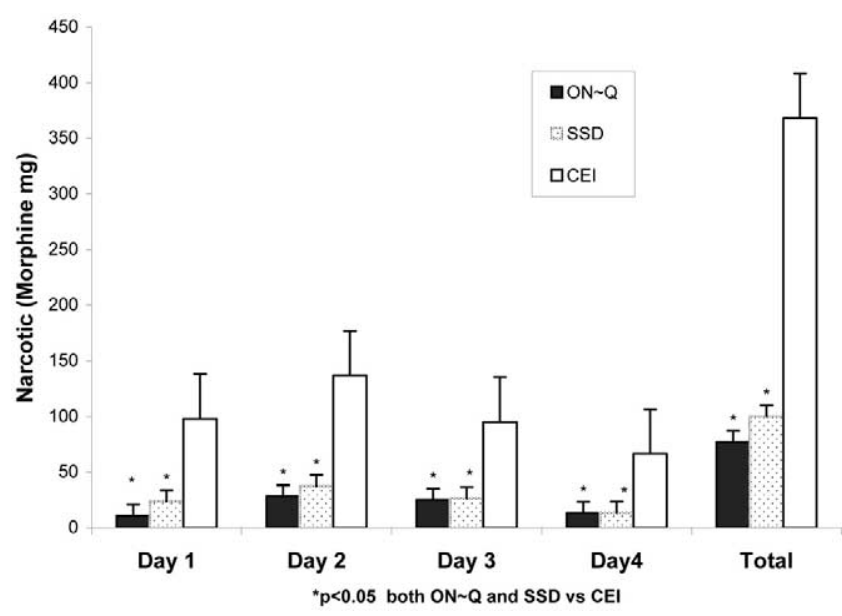

Figure 1. Narcotic use during the first 4 days after surgical intervention and total as assessed by determining morphine equivalency for all narcotics used.
Regional anesthesia techniques, including epidural anesthesia, intercostal nerve blocks, and paravertebral blocks, provide pain relief without the central respiratory depression often associated with narcotics. Epidural anesthesia, a widely used method of controlling postoperative pain after thoracotomy, is considered the gold standard; however, it might not provide adequate relief for all patients. Those patients who have undergone previous operations on the spine or the morbidly obese might not have anatomy suitable for placement of an epidural catheter. Additionally, obese patients have an increased incidence of catheter migration caused by excess skin shifting the catheter position. ${ }^{15}$ Complications associated with epidural analgesia include hypotension, headache, nausea, urinary retention, pruritus, and infection. ${ }^{16,17}$ Epidural management of pain is also labor intensive, requiring resources to titrate dosing and manage the side effects associated with its use.

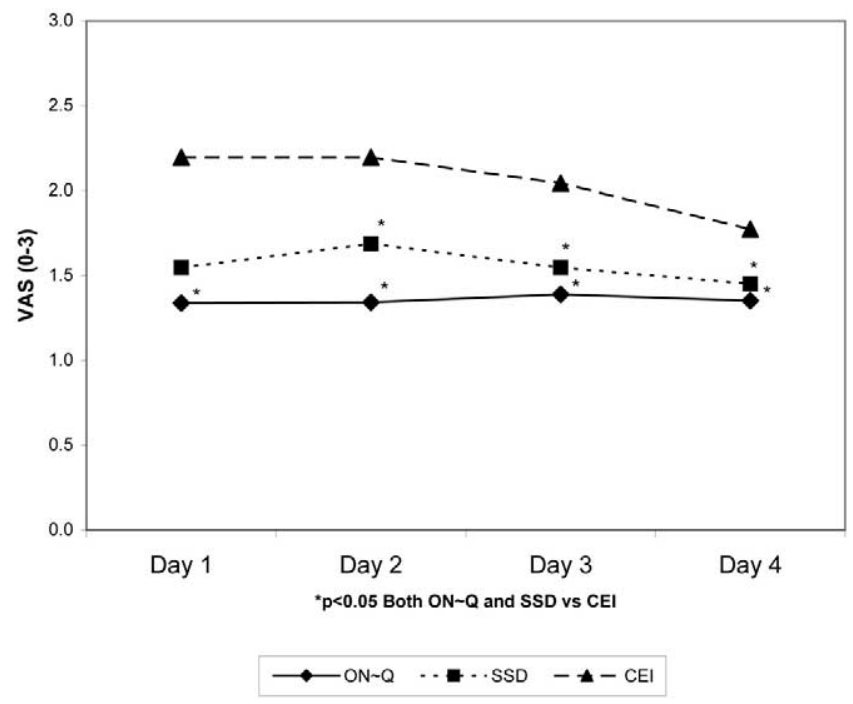

Figure 2. Comparison of VAS pain scores among the 3 pain management groups. 
Local anesthetics have been used for decades in an attempt to control postoperative pain. ${ }^{18}$ Use of continuous infusions of subcutaneous local anesthetics might have the potential to provide superior postoperative analgesia by providing pain relief at the source of injury and avoiding the systemic adverse effects of narcotics. Berrisford and colleagues ${ }^{19}$ described an intercostal continuous nerve block technique that used an indwelling extrapleural catheter that was placed intraoperatively to infuse a local anesthetic in the postoperative period. This strategy demonstrated improved outcomes in several randomized clinical trials. ${ }^{2,20}$ It has been further described in comparative trials that show a significant reduction in narcotic use and decreased pain scores. ${ }^{21,22}$ Patients in these studies did not require treatment for hypotension or catheterization for urinary retention.

Multimodal analgesia has demonstrated increased effectiveness when compared with any single method of reducing pain alone. Numerous studies with regional anesthesia strategies in combination with systemic analgesics have demonstrated improved patient outcomes, including decreased lengths of stay. ${ }^{1,20}$ The use of continuous infusions of local anesthetics with the ON-Q Pain Relief System as part of a multimodal approach to postoperative pain management has been shown to decrease narcotic use, patient's perception of pain, and length of hospital stay in patients undergoing median sternotomy, abdominal hysterectomy, or cesarean section. ${ }^{22-24}$

Additionally, a recent study comparing intercostal nerve catheter-administered local anesthetic and PCA with epidural analgesia demonstrated equivalent pain control but decreased Foley catheter and supplemental narcotic requirements with intercostal nerve catheter-administered local anesthetic and PCA. ${ }^{25}$ The placement of the catheters at the end of the operation is uncomplicated, and its only contraindication is allergy to the local anesthetic. After the operation, the elastomeric pump does not require any adjustment or care by physicians or the nursing staff. Unlike continuous epidurals, which require maintenance, titration, and management of complications, the use of the ON-Q device might lead to a decrease in complications, interventions, and resources needed to treat the patient.

This study demonstrates that the use of the ON-Q device, either alone or with sliding-scale morphine, can reduce a patient's pain after thoracotomy, as measured by the VAS pain score, when compared with use of narcotic epidural infusion alone. This improvement is achieved with less narcotic use because ON-Q reduced total narcotic use by more than half. Improved pain control with less narcotic use might translate into decreased complications, such as hypotension, headache, nausea, urinary retention, pruritus, and infection; however, this study did not assess these outcomes.
We acknowledge the inherent limitations associated with this retrospective review. However, we believe that our careful review of pertinent patient data demonstrates that the use of continuous regional infusion of a long-acting local anesthetic results in less pain and less narcotic use after thoracotomy compared with continuous epidural pain management. A continuous infusion of $0.25 \%$ bupivacaine at $4 \mathrm{~mL} / \mathrm{h}$ through the ON-Q elastomeric infusion pump is a safe and effective adjunct in postoperative pain management for thoracotomy and presents a viable and possibly superior option to continuous epidural anesthesia in patients undergoing this procedure. A randomized prospective evaluation of the ON-Q Pain Relief System should be performed to further elucidate its role in the management of postthoracotomy pain.

We thank Jesse Williams for his insightful suggestions and review of the manuscript. We also acknowledge Rehal Bhojani for his technical assistance.

\section{References}

1. Scott NB, Turfrey DJ, Ray DA, et al. A prospective randomized study of the potential benefits of thoracic epidural anesthesia and analgesia in patients undergoing coronary artery bypass grafting. Anesth Analg. 2001;93:523-5.

2. Deneuvile M, Bisserier A, Regnard J, Chevalier M, Levasseur P, Herve P. Continuous intercostals analgesia with $0.5 \%$ bupivacaine after thoracotomy: a randomized study. Ann Thorac Surg. 1993;55: 381-5.

3. Roberge CW, McEwen M. The effects of local anesthetics on postoperative pain. AORN J. 1998;68:1003-12.

4. Anonymous. Quality improvement guidelines for the treatment of acute pain and cancer pain. JAMA. 1995;274:1874-80.

5. Berrisford RG, Sabanathan S, Mearns AJ, Bickford-Smith PJ. Pulmonary complications after lung resection: the effect of continuous extrapleural intercostals nerve block. Eur J Cardiothorac Surg. 1990;4: 407-11.

6. Ballantyne JC, Carr DB, deFerranti S, et al. The comparative effects of postoperative analgesic therapies on pulmonary outcome: cumulative meta-analyses of randomized, controlled trials. Anesth Analg. 1998; 86:598-612.

7. Sabanathan S, Eng J, Mearns AJ. Alterations in respiratory mechanics following thoracotomy. J R Coll Surg Edinb. 1990;35:144-50.

8. Dahl JB, Moiniche S, Kehlet H. Wound infiltration with local anaesthetics for postoperative pain relief. Acta Anaesthsiol Scand. 1994;38: 7-14.

9. Moiniche S, Mikkelsen S, Wetterslev J, Dahl JB. A qualitative systemic review of incisional local anesthesia for postoperative pain relief after abdominal surgery. Br J Anaesth. 1998;81:377-83.

10. Klein S, Grant SA, Greengrass RA, et al. Interscalene brachial plexus block with a continuous catheter insertion system and a disposable infusion pump. Anesth Analg. 2000;91:1473-8.

11. Carr DB, Jacox AK, Chapman CR, et al. Clinical practice guidelines for acute pain management: operative or medical procedures and trauma. Washington (DC): Agency for Health Care Policy and Research; 1992. DHHS publication no. 95-0034.

12. Dajczman E, Gordon A, Kreisman H, Wolkove N. Long-term postthoracotomy pain. Chest. 1991;99:270-4.

13. Pettersson PH, Lindskog EA, Owall A. Patient-controlled versus nurse-controlled pain treatment after coronary artery bypass surgery. Acta Anaesthesiol Scand. 2000;44:43-7.

14. O'Halloran P, Brown R. Patient-controlled analgesia compared with nurse-controlled infusion analgesia after heart surgery. Intensive Crit Care Nurse. 1997;13:126-9. 
15. Faheem M, Sarwar N. Sliding of the skin over subcutaneous tissue is another important factor in epidural catheter migration. Can J Anaesth. 2002;49:634.

16. Kinsella SM, Tuckey JP. Perioperative bradycardia and asystole: relationship to vasovagal syncope and the Bezold-Jarisch reflex. $\mathrm{Br} J$ Anaesth. 2001;86:859-68.

17. Uitvlugt A. Managing complications of epidural analgesia. Int Anesth Clin. 1990;28:11-6.

18. Chan V, Chung F, Cheng DCH, Kirby TJ Chung A, Sandler A. Continuous intercostal block for pain relief following thoracotomy-a new approach. Can J Anaesth. 1988;35(suppl):S113-4.

19. Berrisford RG, Sabanathan S, Mearns AJ, Clarke BJ. Plasma concentrations of bupivacaine and its enantiomers during continuous intercostal nerve block. Br J Anaesth. 1993;70:201-4.

20. Cheong W, Seow-Choen F, Eu K, Tang C, Heah S. Randomized clinical trial of local bupivacaine perfusion versus parenteral morphine infusion for pain relief after laparotomy. Br J Surg. 2001; 80:519-20.
21. Majid AA, Hamzah H. Pain control after thoracotomy an extrapleural tunnel to provide a continuous bupivacaine infusion for intercostal nerve blockade. Chest. 1992;101:981-4.

22. Barron DJ, et al. A randomized controlled trial of continuous extrapleural analgesia postthoracotomy efficacy and choice of local anesthetic. Eur J Anaesthesiol. 1999;16:236-45.

23. Zimberg S, Davila G, Seiler J, Neimark M. ON-Q Pain Relief System in elective abdominal hysterectomy surgery: a pilot clinical outcomes study evaluating length of stay, postoperative pain, narcotic analgesia use and the impact on costs and adverse effects. Presented at: 50th Annual Clinical Meeting of the American College of Obstetricians and Gynecologists; Los Angeles (CA): May 6, 2002.

24. Givens V, Lipscomb G, Meyer N. A randomized trial of postoperative wound irrigation with local anesthetic for pain after cesarean delivery. Am J Obstet Gynecol. 2002;6:6.

25. Luketich JD, Land SR, Sullivan EA, et al. Thoracic epidural versus intercostal nerve catheter plus PCA morphine: a randomized study. Ann Thorac Surg. In press. 